\title{
Light Guiding by Effective Gauge Field for Photons
}

\author{
Qian $\operatorname{Lin}^{1}$ and Shanhui Fan ${ }^{2}$ \\ ${ }^{1}$ Department of Applied Physics, Stanford University, Stanford, California 94305, USA \\ ${ }^{2}$ Department of Electrical Engineering, Stanford University, Stanford, California 94305, USA \\ (Received 9 April 2014; revised manuscript received 5 August 2014; published 22 August 2014) \\ We propose a waveguiding mechanism based on the effective gauge potential for photons. The \\ waveguide geometry consists of core and cladding regions with the same underlying dispersion relation, \\ but subject to different gauge potentials. This geometry can be realized in a dynamically modulated \\ resonator lattice and provides a conceptually straightforward and dynamically reconfigurable mechanism \\ for generating a one-way waveguide.
}

DOI: 10.1103/PhysRevX.4.031031

\section{INTRODUCTION}

Waveguides are fundamental building blocks of integrated photonics. The most commonly used waveguide consists of a high refractive index core surrounded by a low refractive index cladding [1], as shown in Fig. 1(a). In such a structure, light is guided in the core by total internal reflection. In recent years, there has also been very active research in exploring new mechanisms for waveguiding. Notable examples include photonic band gap guiding [2,3] and waveguides based on surface plasmons [4].

In this paper, we propose an alternative light guiding mechanism based on the concept of an effective gauge field for photons. Photons are neutral particles, and hence there is no naturally existing gauge field that couples to photons. Nevertheless, in recent years, various mechanisms for creating effective gauge field for photons have been proposed [5-14] and demonstrated [15-17]. In particular, it has been pointed out by Fang et al. [18] that a photonic gauge field can be created in a dynamically modulated photonic structure by controlling the modulation phase. Moreover, one effect of such a photonic gauge field is to shift the dispersion relation in the wave vector space [19]. Building upon the proposal in Ref. [19], we show that such a shift can be utilized to construct a waveguide for light based on the effective gauge field for photons [Fig. 1(b)].

The proposed gauge-field waveguide exhibits two major advantages. First of all, it is dynamically reconfigurable. While other waveguides have their structure determined at fabrication, in our proposed waveguide, the dynamic modulation phases can be readily changed, thus allowing the temporal reconfiguration of the waveguide structure. Secondly, dynamically modulated structures exhibit

Published by the American Physical Society under the terms of the Creative Commons Attribution 3.0 License. Further distribution of this work must maintain attribution to the author(s) and the published article's title, journal citation, and DOI.
Subject Areas: Optics, Photonics, Quantum Physics

nonreciprocity, which is absent in other waveguide structures unless magneto-optical or nonlinear materials are used. With the proposed system, we can achieve one-way waveguiding naturally.

The one-way waveguide concept presented here differs from the one-way guiding discussed in Ref. [18] in significant ways. Reference [18] proposes a photonic analog of the integer quantum Hall effect. The one-way guiding there was supported by edge modes, which are located at the surface of a bulk subject to a nonzero effective magnetic field. The edge mode arises from the nontrivial topological properties of the bulk. In contrast, in the present design, both the core and the cladding regions have zero effective magnetic fields and zero Chern numbers. Hence, both the core and the cladding regions by themselves are topologically trivial. The nontrivial one-way guiding arises from the nontrivial topological features of the interfaces between the core and cladding.

Furthermore, in the present design, the guided mode intensity is concentrated at the center of the core region. In contrast, in edge-mode guiding systems [11,12,15-18], the intensity maximum is located at the edge. This difference is of great importance in practice. The entire modern optical information processing system, including both optical fiber networks and on-chip optical interconnect systems, are built upon the kinds of guided modes we propose here. Compared with edge modes, such guided modes are better confined and therefore better insulated from external interferences. Our design therefore represents an important step forward in using one-way waveguide modes in practical informationprocessing circuits.

This paper is organized as follows: in Sec. II, we briefly review the theoretical background, including both the relevant aspects of the standard waveguide theory (Sec. II A) as well as the effects of gauge fields (Sec. II B). In Sec. III, we explain the principle of gauge-field guiding, set up the waveguide theory framework, and analytically solve a simple model to illustrate some general features of such a waveguide. In Sec. IV, we propose a physical system consisting of 
a dynamically modulated resonator lattice where such a gauge-field waveguide for photons may be realized and calculate the waveguide dispersion and some related properties. We conclude in Sec. V.

\section{THEORETICAL BACKGROUND}

\section{A. Relevant aspects of standard waveguide theory}

We start by briefly reviewing relevant aspects of the standard waveguide theory [1]. The standard waveguide theory is applicable to the geometry shown in Fig. 1, where a core region is sandwiched between two cladding regions. For our purpose, it is sufficient to consider only the symmetric waveguide configuration where the two cladding regions are made of the same material. The core and the cladding regions are uniform media with dispersion relation $\omega_{\text {core }}\left(k_{x}, k_{y}\right)$ and $\omega_{\text {cladding }}\left(k_{x}, k_{y}\right)$, respectively, where $k_{y}$ is the wave vector along the waveguide. A guided mode exists if at a given $\left(\omega, k_{y}\right)$ there is a propagating mode in the core that is decoupled from any propagating mode in the cladding. For such a mode, the transverse field profile is exponentially decaying in the cladding region and sinusoidally oscillating in the core region, i.e.,

$$
|\psi\rangle \sim \begin{cases}e^{-\alpha_{x}|x|} & |x|>d / 2 \\ \cos k_{x} x \text { or } \sin k_{x} x & |x| \leq d / 2 .\end{cases}
$$

Here, $k_{x}$ is obtained from the dispersion relation of the core region $\omega_{\text {core }}\left(k_{x}, k_{y}\right)$ and $\alpha_{x}$ is determined by the analytical continuation from the dispersion relation of the cladding $\omega_{\text {cladding }}\left(k_{x}, k_{y}\right)$ into the complex $k_{x}$ plane. Throughout this paper, we define the width of the core region as $d$.

Starting from this field profile in Eq. (1), and assuming that both the field and its derivative are continuous across the core-cladding interface, we obtain the following equation: (a)

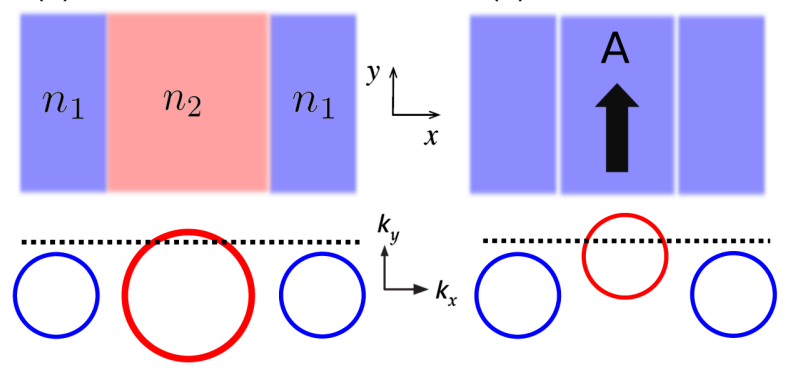

FIG. 1. Structures and isofrequency contours corresponding to two different waveguiding mechanisms: (a) index guiding, where the core region has a high refractive index and the cladding has a low refractive index; (b) gauge-field guiding, where the core region has a nonzero gauge potential $\mathbf{A}$ and the cladding has a zero gauge potential. The dashed line on the isofrequency contours demonstrates the absence of $k_{y}$-matching states in the cladding region.

$$
\alpha_{x}= \begin{cases}k_{x} \tan \left(k_{x} d / 2\right) & \text { even mode } \\ -k_{x} \cot \left(k_{x} d / 2\right) & \text { odd mode }\end{cases}
$$

Solving Eq. (2), we then obtain the mode profile and the dispersion relation of the guided mode.

The derivation above is standard. It was, however, typically carried out to describe the conventional slab waveguide geometry of Fig. 1(a). Here, we reproduce these derivations to emphasize that Eq. (2) applies to slab configurations in general, independent of the form of dispersions of the media in the slab configuration, and, therefore, is applicable to our structure of interest here in Fig. 1(b).

\section{B. Effect of gauge field on dispersion relation}

It is well known in quantum mechanics that to describe the dynamics of an electron in an electromagnetic field one needs to replace the kinetic momentum in the Hamiltonian with the canonical momentum; i.e.,

$$
-i \nabla \rightarrow-i \nabla-\mathbf{A},
$$

Here, we define $\mathbf{A}=q \tilde{\mathbf{A}}$, where $q$ is the charge of the electron and $\tilde{\mathbf{A}}$ is the vector potential for electrons. We choose the unit such that $\hbar=c=1$, and in the following refer to $\mathbf{A}$, rather then $\tilde{\mathbf{A}}$, as the gauge potential, so that the similarity between electronic and photonic gauge fields becomes more transparent. As a result, suppose we have an electronic system described by a dispersion relation $\omega(\mathbf{k})$ in the absence of the gauge potential, where $\omega$ is the frequency and $\mathbf{k}$ is the wave vector of a mode of the system. When a uniform gauge potential $\mathbf{A}$ is applied, its dispersion relation becomes $\omega(\mathbf{k}-\mathbf{A})$; i.e.,

$$
\omega(\mathbf{k}) \rightarrow \omega(\mathbf{k}-\mathbf{A}) .
$$

The effect of gauge field is a shift of the dispersion relation in $k$ space, as specified by A. This applies to electrons in free space and in crystal lattice when subject to a magnetic field.

Photons are neutral particles that do not interact directly with a magnetic field. However, recent theoretical works $[11,12,18,20]$ have shown that an effective magnetic field for photons can exist if light would accumulate a directiondependent phase when propagating. Consequently, the discussion above for electrons applies to photons in an effective gauge field as well: a properly designed photonic gauge field should be able to shift the dispersion relation in the $k$ space. An explicit demonstration of some of the consequences of such a shift has been provided in Ref. [19].

\section{GENERAL PRINCIPLES OF WAVE GUIDING BY GAUGE FIELD}

\section{A. General principles}

Having reviewed the relevant backgrounds, we now consider the geometry in Fig. 1(b). In this geometry, the 
cladding region is described by a material with a dispersion relation $\omega\left(k_{x}, k_{y}\right)$. The core region is described by a material with the same dispersion, but, in addition, subject to a uniform gauge potential along the $y$ direction, i.e., $\mathbf{A}=A_{y} \hat{y}$. Based on the discussion in Sec. II B, the dispersion relation of the core region is then described by $\omega\left(k_{x}, k_{y}-A_{y}\right)$.

The principle of waveguiding in the structure of Fig. 1(b) can be understood by an isofrequency contour analysis [21] analogous to a conventional waveguide. For both slab waveguide structures shown in Fig. 1, the wave vector component $k_{y}$ parallel to the interface is conserved. For conventional index guiding in Fig. 1(a), the core region has a larger isofrequency contour than the cladding region. Consequently, the core supports states that do not have matching states in the cladding region. For our proposed gauge-field waveguide in Fig. 1(b), the isofrequency contours of the core and the cladding regions have the same shape; they are, however, shifted away from each other along the $y$ direction due to an effective gauge field in the core region. As a result, there are now propagating modes in the core region, for example, the modes corresponding to the intersections between the dashed line and the constant frequency contour of the core in Fig. 1(b), which cannot match and couple to the propagating modes in the cladding. Thus, applying a gauge potential along the propagation direction in the core region can create a waveguide based on gauge field.

\section{B. Simple model for gauge-field waveguide}

In this section, we study in detail a simple model system represented by Fig. 1(b) so as to demonstrate the novel properties of the proposed gauge-field waveguide. In the absence of the gauge potential, we assume a linear intrinsic dispersion relation to facilitate comparison with conventional waveguides. The cladding and core are therefore described by

$$
\omega^{2}= \begin{cases}k_{x}^{2}+k_{y}^{2} & \text { cladding region } \\ k_{x}^{2}+\left(k_{y}-A_{y}\right)^{2} & \text { core region. }\end{cases}
$$

The projected band diagrams for the cladding and the core regions are plotted in blue and pink in Figs. 2(b)-2(d). The blue shaded region corresponds to the extended modes in the cladding region, as obtained by projecting the dispersion $\omega^{2}=k_{x}^{2}+k_{y}^{2}$ onto the $\omega-k_{y}$ plane (this shaded region is also referred to as the light cone). The pink shaded region corresponds to the light cone in the core region. The overlapping part of the two light cones is shaded purple.

Solving Eqs. (2) and (3) at different $\omega$ generates the $\omega$ versus $k_{y}$ dispersion relation of the guided mode(s) as shown by the red curves in Figs. 2(b)-2(d). In the plots, we define $\phi \equiv A_{y} d / 2$, which is the only dimensionless constant that carries all the structural information of the gauge-field waveguide system discussed in this section.

A guided mode should be evanescent in the cladding region and propagating in the core region. Therefore, the guided mode should be outside the light cone of the cladding region, and should lie within the light cone of the core region, which we observe in all four dispersion-relation plots. From Fig. 2 alone, we can already conclude some special features of the gauge-field waveguide, many of which are different from the conventional waveguide. (1) Nonreciprocity. For the gauge-field waveguide shown in Figs. 2(b)-2(d), the pink region is shifted to the right along the $k_{y}$ axis by $A_{y}$ with respect to the blue region. Based on what we argued before regarding the position of the guided mode dispersion with respect to the light cones, with $A_{y}>0$, the guided mode can exist only in the region to the right of the cladding light line $\omega=k_{y}$, i.e., with $k_{y}>0$. We indeed observe this in the waveguide mode calculation. In a conventional waveguide system, such as Fig. 2(a), on the other hand, the light cones for both the core and the cladding are symmetric with respect to $k_{y}=0$, and so is the guided mode dispersion, due to time-reversal symmetry.
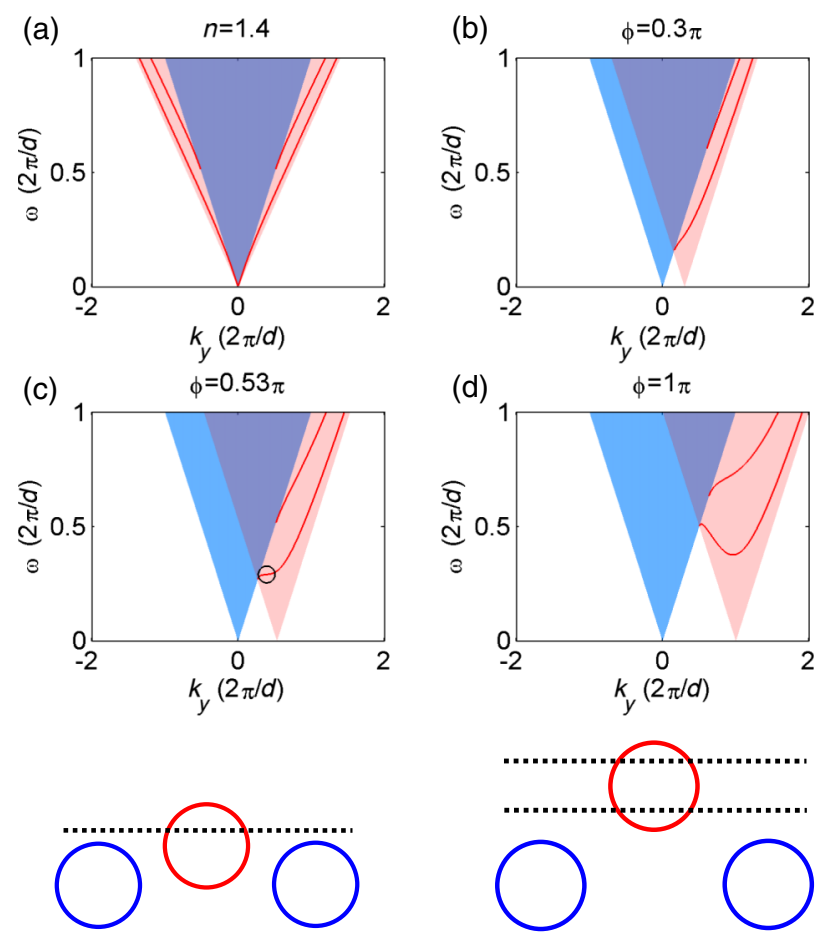

FIG. 2. Dispersion relation of the guided mode (red solid line) for (a) a conventional waveguide with cladding index $n=1$ and core index $n=1.4$, (b) a gauge-field waveguide with $\phi=0.3 \pi$, (c) a gauge-field waveguide with $\phi=0.53 \pi$, with the inflection point [22] marked by a black circle, (d) a gauge-field waveguide with $\phi=\pi$. The pink and blue regions show the light cones for the core and cladding regions, respectively. Isofrequency contours for (e) small and (f) large $\phi$ at the same frequency $\omega$. Panel (e) has only guided mode with $v_{g}>0$ while panel (f) has guided modes with $v_{g}>0$ and $v_{g}<0$. 
Since $A_{y} \neq 0$ breaks time-reversal symmetry, one should certainly expect some effect of nonreciprocity in the waveguide dispersion relation. However, in contrast to many previously considered waveguide systems [8-10], where nonreciprocity is typically manifested in a dispersion with $\omega\left(k_{y}\right) \neq \omega\left(-k_{y}\right)$, the complete absence of guided mode with $k_{y}<0$ is a rather remarkable feature of the gauge-field waveguide system. (2) Single-mode one-way waveguide. Also evident from Figs. 2(b)-2(d), within certain $\omega$ range, for example, the frequency range $\omega \in[0.15,0.6](2 \pi / d)$ in Fig. 2(b), there exists only one guided mode and the slope of the guided mode has a constant sign, signaling the existence of single-mode one-way guiding. Such a single-mode oneway waveguide should provide robust transport that is immune from disorder-induced backscattering [9]. Unlike some of the earlier systems where single-mode one-way waveguide arises from topological features in the bulk of magneto-optical photonic crystal [8-10], the use of the gauge potential provides a simple model where one-way waveguide naturally emerges. (3) Group velocity and inflection point. For small $\left|A_{y}\right|[$ Fig. 2(b)], the group velocity is largely positive $\left(\partial \omega / \partial k_{y}>0\right)$. This is because the isofrequency contours of the core and the cladding regions are still partially overlapping, and only states with a positive group velocity are guided, as shown in Fig. 2(e). On the other hand, for large $\left|A_{y}\right|$ [Fig. 2(d)], the group velocity can be either positive or negative, since the isofrequency contours are shifted sufficiently apart to create guided states with group velocities in both directions, as shown in Fig. 2(f). For one particular $A_{y}$ corresponding to $\phi=0.53 \pi$, there is a stationary inflection point in the guided mode dispersion with $\partial \omega / \partial k_{y}=0, \partial^{2} \omega / \partial k_{y}^{2}=0$, and $\partial^{3} \omega / \partial k_{y}^{3} \neq 0$, shown by the black circle in Fig. 2(c). As discussed in Ref. [22], such a point is of great interest for slow light applications. (4) Cutoff in the fundamental mode. In waveguide theory, the cutoff of a mode is defined as the frequency where the guided mode emerges from the cladding region light cone. Examining the light cones in Figs. 2(b)-2(d), we observe that the cutoff has to have a nonzero frequency, including the cutoff for the fundamental mode. The reason being that there is no phase space of the core light cone available around the cladding light line for $\omega<\left|A_{y}\right| / 2$. This is again a feature not seen in a conventional waveguide. In a conventional waveguide, the core light cone encompasses the cladding light cone, so the fundamental mode can emerge into the core light cone starting from $\omega=0$, as shown in Fig. 2(a).

\section{PROPOSAL FOR REALIZING GAUGE-FIELD WAVEGUIDE}

The simple model discussed in the previous section demonstrates many novel waveguide features. However, it is a nontrivial task to generate an effective gauge field for photons [5-10,18]. In this section, we propose a realization of optical wave guiding by an effective gauge field.
The physical system is an array of dynamically coupled resonant cavities where the nearest-neighbor couplings are modulated by active refractive index modulations with controlled modulation phases. First, we briefly summarize the relevant results of Ref. [18], which has shown that the modulation phase emerges as an effective gauge potential in a square lattice of coupled resonators. Then, we define a waveguide geometry in this system and analytically calculate the band structure and wave function of the guided mode using the real-system Hamiltonian and compare the result with our waveguide theory analysis.

\section{A. Effective gauge field for photons with dynamic modulation}

To achieve a photonic gauge field in a physical model, we follow the proposal of Fang et al. in Ref. [18]. Consider that a lattice consists of two interpenetrating sets of resonators with resonant frequencies $\omega_{A}$ and $\omega_{B}\left(\omega_{A}<\omega_{B}\right)$. The spacial separation between nearest neighbors is $a$. Assume that the nearest-neighbor coupling is achieved by a time-dependent harmonic index modulation with frequency $\Omega$ and coupling strength $V$. The Hamiltonian describing the system is

$$
\begin{aligned}
H= & \omega_{A} \sum_{i} a_{i}^{\dagger} a_{i}+\omega_{B} \sum_{j} b_{j}^{\dagger} b_{j} \\
& +\sum_{\langle i j\rangle} V \cos \left(\Omega t+\phi_{i j}\right)\left(a_{i}^{\dagger} b_{j}+b_{j}^{\dagger} a_{i}\right) .
\end{aligned}
$$

Assume the modulation is on resonance; i.e., $\Omega=\omega_{B}-\omega_{A}$. In the limit $V \leq \Omega$, we can use the rotating-wave approximation to transform the Hamiltonian of Eq. (4) into

$$
H_{\mathrm{RWA}}=\sum_{\langle i j\rangle} \frac{V}{2}\left(e^{i \phi_{i j}} c_{i}^{\dagger} c_{j}+e^{-i \phi_{i j}} c_{j}^{\dagger} c_{i}\right),
$$

where $c_{i(j)}=e^{i \omega_{A(B)} t} a_{i}\left(b_{j}\right)$. Notice that if all $\phi_{i j}=0$, Eq. (5) describes a simple tight-binding model on a square lattice, with spatial periodicity $a$ in both the $x$ and $y$ directions.

Comparing Eqs. (4) and (5), we see that the modulation phase $\phi_{i j}$ gives rise to a direction-dependent hopping term between nearest neighbors and generates an effective gauge potential for photons. The strength of the gauge potential at a bond $\langle i j\rangle$ is related to $\phi_{i j}$ by

$$
\int_{i}^{j} \mathbf{A} \cdot d \mathbf{l}=\phi_{i j}
$$

Figure 3 shows the projected band diagram calculated from Eq. (5) for two different $\left\{\phi_{i j}\right\}$ configurations. The shaded regions here correspond to the extended states in the lattice. Therefore, drawing analogy to the case discussed in Sec. II, we refer to these shaded regions as "light cones" as well, even though these regions are no longer of a cone 

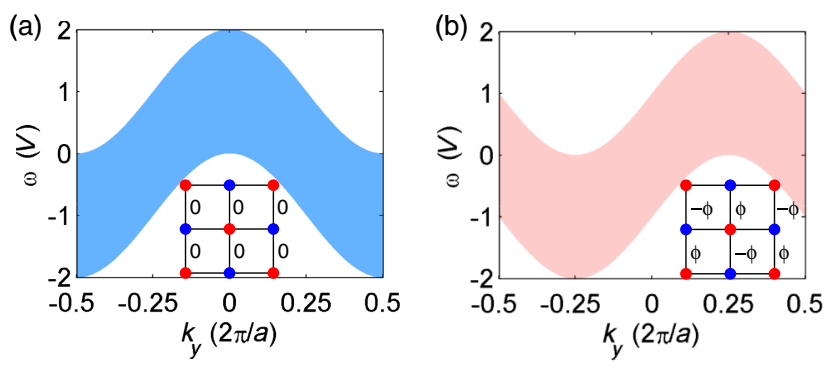

FIG. 3. Projected band diagrams for a modulated square lattice (a) with no gauge field and (b) with an effective gauge field of $\phi=0.5 \pi$. The insers show the lattice configuration with the corresponding modulation phases.

shape. Figure 3(a) corresponds to zero gauge field everywhere. In this case, the dispersion relation of the lattice is

$$
\omega=V\left(\cos k_{x} a+\cos k_{y} a\right) .
$$

Figure 3(a) is the projection of such a dispersion relation onto the $\omega-k_{y}$ plane. Figure 3(b) corresponds to uniform $\mathbf{A}=(\phi / a) \hat{y} \equiv A_{y} \hat{y}$. The dispersion relation is

$$
\omega=V\left[\cos k_{x} a+\cos \left(k_{y} a-\phi\right)\right] .
$$

Figure 3(b) is the projection of this dispersion relation onto the $\omega-k_{y}$ plane. Comparing Fig. 3(a) with Fig. 3(b), we see that the effect of the gauge potential is a shift of the light cone along the $k_{y}$ axis by $A_{y}$.

\section{B. Implementing gauge-field fuiding in dynamically coupled resonator arrays}

Building upon the resonator model above, we now construct a waveguide. Consider a strip of resonator lattice finite in $x$ (lateral confinement direction) and infinite in $y$ (wave propagating direction). The modulation phase distribution [Fig. 4(a)] is such that an effective gauge field $\mathbf{A}=A_{y} \hat{y}=(\phi / a) \hat{y}$ is present in the central $N$ columns (core region with $d=N a$ ), as shown in Fig. 4(b), while being uniformly zero on both sides (cladding region). The cladding region is assumed to be much wider than the core region. This configuration provides an implementation of our conceptual system in Fig. 1(b).

Figure 4(b) can be characterized by a set of directional coupling phases $\left\{\phi\left(x_{n}\right)\right\}$, which is only a function of the $x$ position. $\phi\left(x_{n}\right)=0$ when $x_{n}$ is in the cladding region and $\phi\left(x_{n}\right)=\phi \neq 0$ when $x_{n}$ is in the core region. Since the system preserves translational symmetry in the $y$ direction, $k_{y}$ is a good quantum number. Thus, the Hamiltonian in Eq. (5) is block diagonalized. In each subspace characterized by $k_{y}$, we can write out the Hamiltonian in the $x$-position base $\left|x_{n}\right\rangle$ :
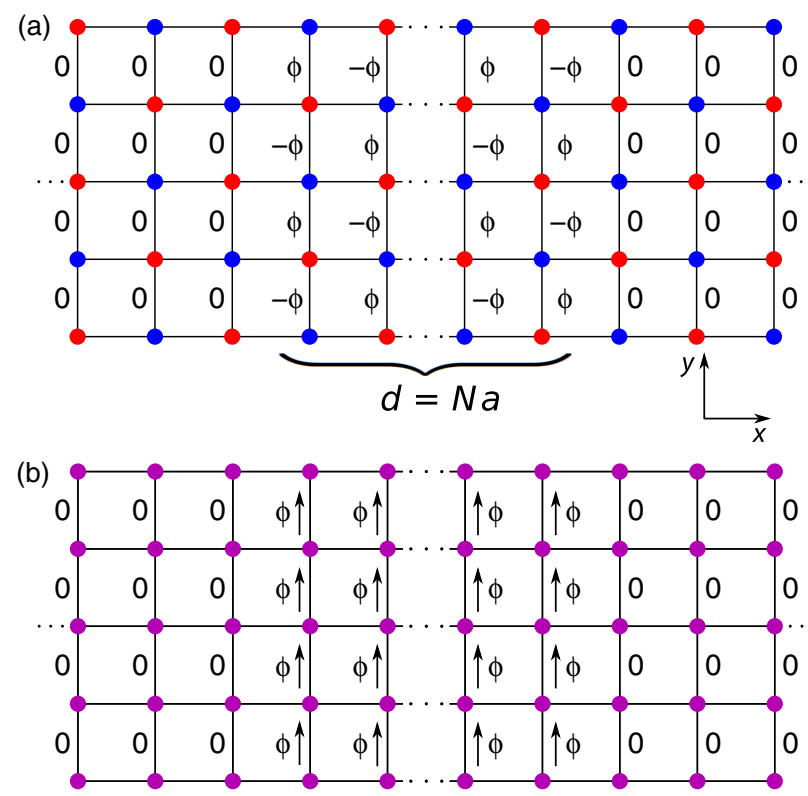

FIG. 4. (a) A square lattice of resonators with resonant frequencies $\omega_{A}$ (red) and $\omega_{B}$ (blue) described by the timedependent Hamiltonian of Eq. (4). The label on each vertical bond denotes the phase of the dynamic modulation between nearest neighbors along the $y$ direction. All the horizontal couplings have a zero modulation phase. (b) A square lattice of identical resonators with nearest-neighbor couplings described by Eq. (5), which is obtained after performing the rotating wave approximation on Eq. (4). In the core region, there is a directiondependent coupling phase $(+\phi$ hopping upward and $-\phi$ hopping downward), which corresponds to a uniform gauge potential. Thus, under rotating wave approximation, the modulation phase in (a) is mapped into a time-independent gauge potential in (b).

$$
\begin{aligned}
H\left(k_{y},\left\{\phi\left(x_{n}\right)\right\}\right)= & V \sum_{n} \cos \left[k_{y} a-\phi\left(x_{n}\right)\right] c_{x_{n}, k_{y}}^{\dagger} c_{x_{n}, k_{y}} \\
& +\frac{V}{2} \sum_{n}\left(c_{x_{n+1}, k_{y}}^{\dagger} c_{x_{n}, k_{y}}+c_{x_{n}, k_{y}}^{\dagger} c_{x_{n+1}, k_{y}}\right) .
\end{aligned}
$$

It is straightforward to solve for the eigenstates and eigenvalues of the Hamiltonian in Eq. (6) and obtain the band structure. Figure 5 shows the result for $d=2 a$ and a cladding width of $10 a$ on each side.

We immediately observe several features that are reminiscent of the waveguide dispersion relation for our simple model in Sec. II. (1) The guided modes exist in the light cone of the core region (pink regions in Fig. 5), and lie completely outside the light cone of the cladding region (blue regions in Fig. 5). For any nonzero value of $A_{y}$, the structure always supports a fundamental mode. As a rather curious feature, the dispersion relation of the fundamental mode always connects between two points where the boundaries of the two light cones intersect. In Sec. IV C, we account for this feature analytically using waveguide theory. For values of $\phi$ near 0 or $2 \pi$, the difference between the core and the cladding light cones is relatively small, 

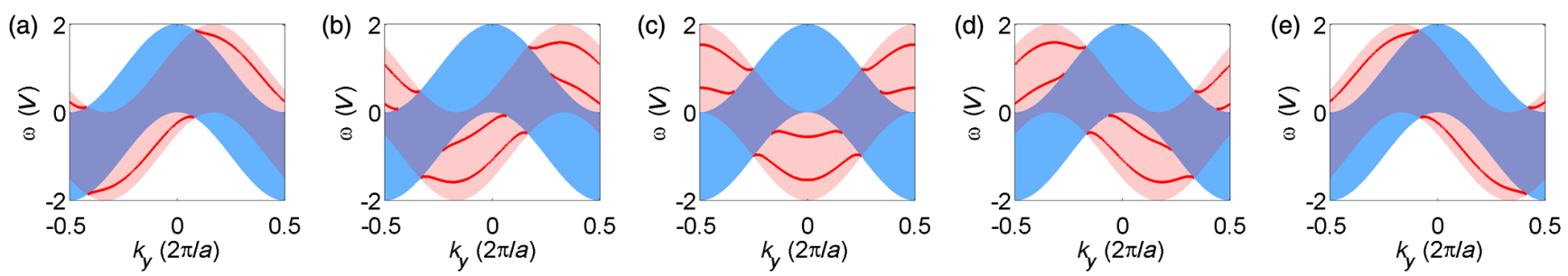

FIG. 5. Guided mode dispersions obtained by solving the Hamiltonian in Eq. (6) with a core region width $d=2 a(N=2)$ and a cladding region width of $10 a$ on each side for $\phi=A_{y} a=[1 / 3,2 / 3,1,4 / 3,5 / 3] \pi$ (a)-(e).

and the structure supports only a fundamental mode. For values of $\phi$ near $\pi$, the core and cladding light cones significantly differ, and higher-order modes could emerge. (2) Nonreciprocity. With the exception of $\phi=0$ and $\phi=\pi$, the dispersion relation is nonreciprocal, i.e., $\omega\left(k_{y}\right) \neq$ $\omega\left(-k_{y}\right)$. (3) Single-mode one-way guiding. For $\phi \neq 0$ and $\phi \neq \pi$, the guided mode dispersion exhibits one-way guiding behavior in certain frequency ranges. This results from a combination of nonreciprocity and the dispersion relation being monotonic in certain frequency ranges. The frequency range for single-mode one-way guiding is particularly large for the cases with smaller $\left|A_{y}\right|$, where the structure supports only the fundamental mode. In such a case, the system provides single-mode one-way guiding for almost the entire range of frequencies where the system supports a guided mode. Thus, many of the aspects we highlighted in Sec. III B using a simple model for a gauge-field waveguide should, in fact, be quite general in different gauge-field waveguides.

There are, on the other hand, some aspects of the waveguide dispersion relation in the present system that are uniquely related to the discrete nature of the lattice. Most notably, the spatial quantization introduces a periodicity in the wave vector space, and a periodicity in $A_{y}$ as well. A change of $2 \pi$ for $\phi=A_{y} a$ corresponds to a change of the magnetic flux through a unit cell by one flux quanta, for which the Hamiltonian is unchanged. Also, at $\phi=\pi$, the Hamiltonian is real with no time-reversal symmetry breaking. The resulting guided mode dispersion at $\phi=\pi$ is, therefore, symmetric.

From Fig. 5, we also observe some prominent symmetries of the guided mode dispersion and the corresponding wave function. (1) The guided mode dispersion for two systems subject to opposite gauge potentials is related by $\omega\left(A_{y}, k_{y}\right)=\omega\left(-A_{y},-k_{y}\right)$. (2) At every value of the gauge field, the guided mode dispersion has the relation $\omega\left(k_{y}+\pi / a\right)=-\omega\left(k_{y}\right)$. The wave functions of the two corresponding eigenstates differ by an alternating sign in their $\left|x_{n}\right\rangle$ coefficients, as shown in Fig. 6. These symmetries are embedded in the Hamiltonian in Eq. (6).

In fact, these symmetries exist not only for our specific gauge-field configuration in Fig. 4, but also for any $\left\{\phi\left(x_{n}\right)\right\}$ (i.e., any modulation phase distribution in the Landau gauge), since the Hamiltonian in Eq. (6) itself describes a generic Landau gauge system.

\section{Comparison with waveguide theory}

Here, we compare the results of exact numerical simulation of the tight-binding model, with the standard waveguide theory as discussed in Sec. II B, where the core and cladding regions are described in terms of their bulk dispersion relations. Such a standard waveguide theory ignores some of the discrete nature of the lattice model. Strictly speaking, the bulk dispersion relations of the core and cladding regions are applicable only when the width of the core and cladding regions are both infinite. Nevertheless, the agreement with the exact calculation based on the tightbinding model is remarkably good even down to a core region width $d=a(N=1)$, as shown in Fig. 7 .

Furthermore, the waveguide theory also provides a simple analytical expression for the cutoff $\phi_{c}$ (which represents a cutoff in the gauge field) for higher-order modes, above which a higher-order mode emerges. At cutoff $\alpha_{x}=0$, the $s$ th-order mode thus has $k_{x}=(s-1)(\pi / N)$, and hence the cutoff condition

$$
\phi_{c}=2 \arcsin \left( \pm \sin ^{2} \frac{(s-1) \pi}{2 N}\right), \quad s \leq N .
$$

This result predicts nonzero cutoffs for higher-order modes with $s>1$. For $s=1$, it predicts $\phi_{c}=0$, which confirms our observation in Sec. IV B that a fundamental mode exists for any nonzero $A_{y}$. The theoretical result is plotted in Fig. 8 together with the numerical results from the tight-binding model. The two sets show excellent agreement. Therefore, in general, one can use the standard waveguide theory to
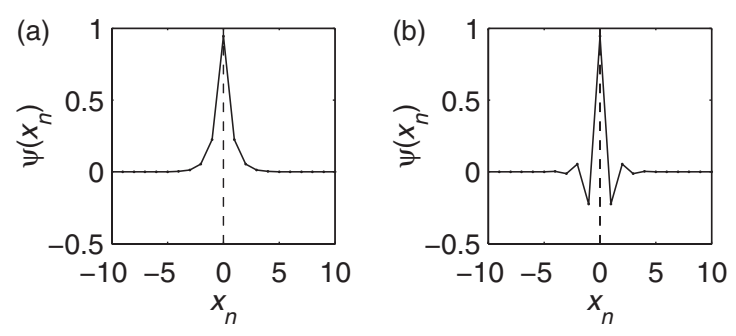

FIG. 6. Guided mode wave function (amplitudes as a function of $x_{n}$ ) for $\phi=\pi$ at (a) the highest band at $k_{y}=\pi / a$ and (b) the lowest band at $k_{y}=0$. The calculation is based on $N=1$ in the core region and $10 a$ on each side in the cladding region. 

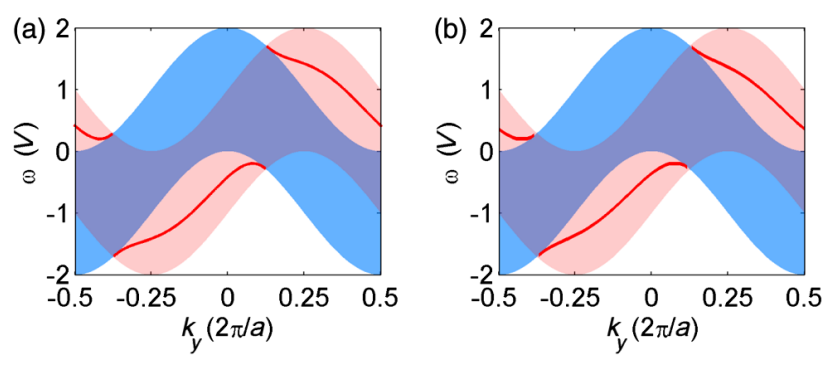

FIG. 7. Guided mode dispersion for $\phi=0.5 \pi$ and $N=1$ obtained by (a) numerically solving the tight-binding model Hamiltonian in Eq. (6) with cladding region width of $10 a$ on each side and (b) waveguide theory calculation.

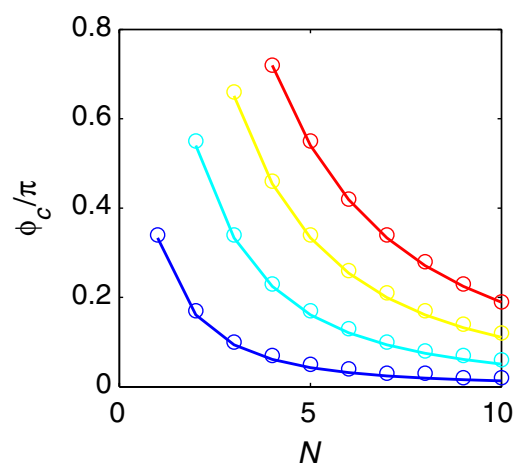

FIG. 8. Cutoff $\phi_{c}$ for higher-order modes when $N \geq 2$. The mode order varies from $s=2$ (in blue) to $s=5$ (in red). Circles represent numerical results from tight-binding model. Curves represent analytical results from waveguide theory.

understand various aspects of the gauge-field waveguide implemented in a lattice model.

The waveguide theory also provides an analytical explanation for the following feature observed in Fig. 5: the dispersion relation of the fundamental mode always connects between two points where the boundaries of the two light cones intersect. We prove this by explicitly constructing the solution for Eq. (2). Notice that at the intersect we have $k_{y} a=\phi / 2$ or $k_{y} a=\phi / 2-\pi$. For the upper band, the analytical continuation is from $\left(k_{x}, k_{y}\right)=(0,0)$, so the intersect has $\alpha_{x}=0$ in the cladding and $k_{x}=0$ in the core, with $\omega=1 \pm \cos (\phi / 2)$. It is easy to verify that this set of parameters satisfies both the cladding and core dispersion relations shown in Sec. IVA, as well as Eq. (2). Similarly, for the lower band, the analytical continuation is from $\left(k_{x}, k_{y}\right)=(\pi / a, \pi / a)$, so the intersect has $\alpha_{x}=0$ in the cladding and $k_{x}=\pi / a$ in the core, with $\omega=-1 \pm \cos (\phi / 2)$. This set of parameters also satisfies all the relevant equations.

\section{FINAL REMARKS AND CONCLUSION}

As a final remark, we comment on the experimental feasibility of the theoretical proposal. The feasibility of the experimental demonstration of the Hamiltonian of Eq. (5) has been discussed extensively in Ref. [18] for both rf and optical frequency ranges. As a very important first step towards experimentally realizing the Hamiltonian of Eq. (5), the existence of a photonic gauge potential has been proven through the experimental realization of the photonic Aharonov-Bohm effect for rf [23] and optical frequency photons [24,25]. In Refs. [23-25], the gauge potential for photons was demonstrated in systems with two mixers or modulators, where the local oscillator phases at each mixer or modulator were controlled to provide the photonic gauge potential. To demonstrate the Hamiltonian of Eq. (5) requires integration and control of a larger number of mixers or modulators as compared to what has been used in Refs. [23-25]. We do think that such a task should be feasible, given the state-of-the-art microwave circuits and the rapid development of large-scale silicon-integrated photonic circuits. Once a scalable system with individually controllable coupling modulation is conceived, a rich system of arbitrarily engineered effective gauge fields can be realized, providing the potential to explore a wider range of phenomena in gauge-field physics that is difficult to realize in solidstate systems. We also note that similar gauge-field concepts should be experimentally implementable in the optical analogs of topological insulators, where a gauge potential depending on photon spin has been theoretically proposed and experimentally realized $[12,13,15,16,26]$.

In summary, we present a new kind of waveguide realized by an inhomogeneous effective gauge field in a square lattice of photonic crystal resonators. Furthermore, we account for the properties of such a waveguide by extending the standard waveguide theory. The gauge-field waveguide provides a very rich set of novel nonreciprocal waveguide features.

\section{ACKNOWLEDGMENTS}

This work is supported in part by U.S. Air Force Office of Scientific Research Grant No. FA9550-09-1-0704 and U.S. National Science Foundation Grant No. ECCS1201914.

[1] H. A. Haus, Waves and Fields in Optoelectronics (Prentice-Hall, Englewood Cliffs, 1984), Chap. 6.

[2] J. D. Joannopoulos, P. R. Villeneuve, and S. Fan, Photonic Crystals: Putting a New Rwist on Light, Nature (London) 386, 143 (1997).

[3] P. Russell, Photonic Crystal Fibers, Science 299, 358 (2003).

[4] S. A. Maier and H. A. Atwater, Plasmonics: Localization and Guiding of Electromagnetic Energy in Metal/Dielectric Structures, J. Appl. Phys. 98, 011101 (2005).

[5] M. Onoda, S. Murakami, and N. Nagaosa, Hall Effect of Light, Phys. Rev. Lett. 93, 083901 (2004). 
[6] S. Raghu and F. D. M. Haldane, Analogs of Quantum-HallEffect Edge States in Photonic Crystals, Phys. Rev. A 78, 033834 (2008).

[7] F. D. M. Haldane and S. Raghu, Possible Realization of Directional Optical Waveguides in Photonic Crystals with Broken Time-Reversal Symmetry, Phys. Rev. Lett. 100, 013904 (2008).

[8] Z. Wang, Y. D. Chong, J. D. Joannopoulos, and M. Soljačić, Reflection-Free One-Way Edge Modes in a Gyromagnetic Photonic Crystal, Phys. Rev. Lett. 100, 013905 (2008).

[9] Z. Wang, Y. D. Chong, J. D. Joannopoulos, and M. Soljačić, Observation of Unidirectional Backscattering-Immune Topological Electromagnetic States, Nature (London) 461, 772 (2009).

[10] Z. Yu, G. Veronis, Z. Wang, and S. Fan, One-Way Electromagnetic Waveguide Formed at the Interface between a Plasmonic Metal under a Static Magnetic Field and a Photonic Crystal, Phys. Rev. Lett. 100, 023902 (2008).

[11] R. O. Umucalilar and I. Carusotto, Artificial Gauge Field for Photons in Coupled Cavity Arrays, Phys. Rev. A 84, 043804 (2011).

[12] M. Hafezi, E. A. Demler, M. D. Lukin, and J. M. Taylor, Robust Optical Delay Lines with Topological Protection, Nat. Phys. 7, 907 (2011).

[13] A. B. Khanikaev, S. H. Mousavi, W.-K. Tse, M. Kargarian, A. H. MacDonald, and G. Shvets, Photonic Topological Insulators, Nat. Mater. 12, 233 (2013).

[14] S. Longhi, Effective Magnetic Fields for Photons in Waveguide and Coupled Resonator Lattices, Opt. Lett. 38, 3570 (2013).

[15] M. C. Rechtsman, J. M. Zeuner, A. Tunnermann, S. Nolte, M. Segev, and A. Szameit, Strain-Induced Pseudomagnetic Field and Photonic Landau Levels in Dielectric Structures, Nat. Photonics 7, 153 (2013).
[16] M. C. Rechtsman, J. M. Zeuner, Y. Plotnik, Y. Lumer, D. Podolsky, F. Dreisow, S. Nolte, M. Segev, and A. Szameit, Photonic Floquet Topological Insulators, Nature (London) 496, 196 (2013).

[17] M. Hafezi, S. Mittal, J. Fan, A. Migdall, and J. M. Taylor, Imaging Topological Edge States in Silicon Photonics, Nat. Photonics 7, 1001 (2013).

[18] K. Fang, Z. Yu, and S. Fan, Realizing Effective Magnetic Field for Photons by Controlling the Phase of Dynamic Modulation, Nat. Photonics 6, 782 (2012).

[19] K. Fang and S. Fan, Controlling the Flow of Light Using the Inhomogeneous Effective Gauge Field that Emerges from Dynamic Modulation, Phys. Rev. Lett. 111, 203901 (2013).

[20] K. Fang, Z. Yu, and S. Fan, Photonic Aharonov-Bohm Effect Based on Dynamic Modulation, Phys. Rev. Lett. 108, 153901 (2012).

[21] J. D. Joannopoulos, S. G. Johnson, J. N. Winn, and R. D. Meade, Photonic Crystals: Molding the Flow of Light, 2nd ed. (Princeton University Press, Princeton, 2011), Chap. 10.

[22] A. Figotin and I. Vitebsky, Nonreciprocal Magnetic Photonic Crystals, Phys. Rev. E 63, 066609 (2001).

[23] K. Fang, Z. Yu, and S. Fan, Experimental Demonstration of a Photonic Aharonov-Bohm Effect at Radio Frequencies, Phys. Rev. B 87, 060301 (2013).

[24] L. D. Tzuang, K. Fang, P. Nussenzveig, S. Fan, and M. Lipson, Non-Reciprocal Phase Shift Induced by an Effective Magnetic Flux for Light, Nat. Photonicics, doi:10.1038/ nphoton.2014.177 (2014).

[25] E. Li, B. J. Eggleton, K. Fang, and S. Fan, Photonic Aharonov-Bohm Effect in Photon-Phonon Interactions, Nat. Commun. 5, 3225 (2014).

[26] M. Pasek and Y. D. Chong, Network Models of Photonic Floquet Topological Insulators, Phys. Rev. B 89, 075113 (2014). 